\title{
同期经尿道等离子电切术治疗膀胱癌合并前列腺增生的 价值分析
}

\author{
哈华晶* 杨立军 李炳义 \\ 渭南市第二医院 陕西 渭南 714000
}

摘 要: 目的: 就同期经尿道等离子点切术对于膀胱癌合并前列腺增生的治疗效果进行了探究。方法: 对本院收 治的64例膀胱癌合并前例腺增生患者作为研究对象, 所有患者均给予同期经尿道等离子点切术进行治疗, 就患者治疗 效果进行分析。结果: 所有患者手术均顺利进行, 术后前例腺症状均得到了显著的改善 $(P<0.05)$ 术后对患者进行 为期 12 月的随访, 有 4 例患者出现了复发情况, 1 例患者因为膀胱癌全身转移出现死亡。结论: 通过同期经尿道等离子 电切术能够对膀胱癌合并前列腺增生患者起到良好的治疗效果，其治疗安全性高，值得临床应用推广。

关键词：同期经尿道等离子电切术；膀胱癌；前列腺增生

DOI: https://doi.org/10.37155/2717-5669-0203-8

前列腺增生属于常见临床疾病, 多发于老年患者, 而膀胱癌的发病率也呈现出了越来越高的趋势。膀胱癌合并前 列腺增生不仅会严重影响患者的生存质量, 也会对患者的生命安全造成一定的威胁。目前临床上认为对于膀胱癌合并 前列腺增生患者需要尽早接受手术治疗。经尿道等离子电切术及膀胱肿瘤部分切除术均是常用的术式, 本研究旨在探 讨二者的效果。

\section{1 资料与方法}

\section{1 一般资料}

本次研究中选取2020年1月～2020年9月期间的64例膀胱癌合并前列腺增生患者作为研究对象，患者年龄在59 78 岁, 平均年龄为 $(65.4 \pm 5.9)$ 岁，病程时间为 5 个月 5年, 平均病程时间 $(2.9 \pm 0.6)$ 年。所有患者在术前均行 CT、 肚门指检以及B超等检查, 并且均被确诊。通过B超进行患者前例腺重量的测量工作, 测定结果为 $36 \sim 77 \mathrm{~g}$, 平均重量 为 $(54.9 \pm 9.1) \mathrm{g}$ 。此外在 64 例患者中, 无蒂 10 例, 有蒂 54 例。所有患者对本次实验均知情, 并且进行了知情同意书 的签署。

\section{2 方法}

本组患者均采取同期经尿道等离子电切术治疗, 术前伴糖尿病者, 给予降糖治疗, 伴尿路感染控制感染, 伴心肺 肾疾病患者行功能改善。应用等离子双极电切系统 (英国Gyrus公司), $360^{\circ}$ 旋转连续冲洗式F 27 镜鞘, $30^{\circ}$ 观察镜, 电 切输出功率与电凝功率分别为 $160 、 80 \mathrm{~W}$, 冲洗液使用生理盐水, 灌注压力为 $60 \mathrm{cmH}_{2} \mathrm{O}$ 。连续硬膜外麻醉, 取患者截 石位, 电切镜经尿道置人, 对其尿道括约肌、双输尿管开口等情况进行观察, 了解患者肿瘤位置、大小和输尿管开口 之间的关系 ${ }^{[1]}$ 。首先采取经尿道膀胱肿瘤切除术, 将肿瘤与其基底周围 $2 \mathrm{~cm}$ 膀胱黏膜切除, 深至肌层, 在止血以后, 使用ELLIK将肿瘤组织碎片冲吸出来, 送检。然后采取经尿道前列腺切除术, 行分区切除, 在6处切一标志沟达包膜 止于精阜, 将左右两侧叶分别切除, 再将12点处顶部前列腺组织切除, 最后将前列腺尖部与颈口切除, 止血, 使用 ELLIK将前列腺碎块吸出。观察患者膀胱, 防止肿瘤遗留, 将F20或者是F 22 气囊导尿管留置。术后1 3 d使用生理盐水 进行冲洗, 硬膜外镇痛洜留置, 持续 $48 \mathrm{~h}$ 镇痛。术后1周, 使用 $40 \mathrm{ml}$ 注射用水加 $30 \mathrm{mg}$ 吡柔比星进行膀胱灌注, 持续灌 注6次, 之后1次/月，持续灌注6次，定期复查 ${ }^{[2]}$ 。

1.3 统计学方法

在软件SPSS19.0中对数据进行处理分析, 计数资料和计量资料分别进行 $\%$ 和 $(\bar{x} \pm s)$ 表示, 进行域者检验, 若是

*通讯作者: 哈华晶, 男, 汉族, 1985.11.20, 甘肃兰州, 本科, 主治医师。研究方向: 老年男性前列腺疾病及泌 尿系结石治疗。 
组间有差异性，则 $P<0.05$, 有统计学意义。

\section{2 结果}

2.1 患者治疗前后前列腺症状改善情况

就所有患者治疗前后最大尿流量、残余尿量以及前列腺症状评分等指标进行对比分析, 发现所有患者治疗后的前 例腺评价指标得到了显著的改善 $(P<0.05)$ ，见表1。

表1 患者在治疗前后前列腺症状改善情况对比

\begin{tabular}{|c|c|c|c|c|}
\hline 组别 & $n$ & 最大尿流量 $(\mathrm{ml} / \mathrm{s})$ & 残留尿量 $(\mathrm{ml})$ & 国际前例腺症状评分 \\
\hline 治疗前 & 32 & $7.1 \pm 3.8$ & $157.3 \pm 53.4$ & $24.8 \pm 4.3$ \\
\hline 治疗后 & 32 & $19.1 \pm 4.6$ & $26.3 \pm 9.8$ & $6.2 \pm 3.6$ \\
\hline$t$ & & 16.089 & 19.303 & 26.533 \\
\hline$P$ & & 0.000 & 0.000 & 0.000 \\
\hline
\end{tabular}

2.2 患者随访情况

所有患者的手术均顺利进行，手术时间控制在50 100min，平均手术时间（83.1 \pm 6.9） min。对患者进行为期12月 的随访，其中有 4 例患者出现了复发情况，并且有 1 例患者因为膀胱癌全身转移出现死亡。

\section{3 讨论}

前列腺增生作为临床上较为常见的一种疾病, 多发于中老年性, 临床症状表现为尿失禁、尿急以及排尿困难等诸 多症状，对于患者的日常生活也会造成非常大的威胁。若未曾进行前列腺的及时治疗，会让患者因为长时间排尿困难 而导致尿液中残留化学成分等问题, 严重情况下还会直接威胁到患者的生命安全。膀胱癌作为尿路上皮肿瘤中的一种 常见肿瘤, 该病症多表现为表浅性肿瘤, 跟合并前列腺增生的发病率甚至达到了 $8 \%$, 目前多是通过手术模式来进行 治疗。临床医学上对于膀胱癌合并前列腺增生能否进行同期手术还有着非常大的争议性, 部分学者认为同期进行前列 腺以及膀胱肿瘤的切除工作是, 还可能导致肿瘤前列腺种植等情况发生, 导致肿瘤的复发率得到进一步的提升, 因此 适合通过分期手术来进行治疗。

随着我国医疗技术的不断发展, 对于前列腺肿瘤合并膀胱癌的研究力度得到了进一步的提升, 发现同期手术很少 会出现膀胱肿瘤种植在前列腺中的形式, 并发症发生的机率也相对比较低。前例腺增生导致尿路梗阻也是导致膀胱 肿瘤发生的一个重要因素, 患者在长时间前例腺增生时会导致尿中致癌物质跟膀胱黏膜接触时间过长, 使得膀胱肿瘤 的发生率得到进一步的提升。此外尿路梗阻还会导致患者出现尿路感染以及膀胱结石等问题, 进一步发展为鳞癌跟腺 癌等问题。在对膀胱癌合并前列腺增生患者进行治疗时, 还需要积极进行前列腺增生的治疗, 这样也就可以进行梗阻 的有效解除, 促使肿瘤复发率得以降低。在本次研究中的 64 例膀胱癌合并前列腺增生患者中, 仅仅出现了4例并发患 者, 而且复发位置均位于前列腺窝以及后尿道中, 也就表明了同期手术不会导致患者的复发率增大, 并且能够具备有 良好的治疗效果 ${ }^{[3]}$ 。

等离子双极电切系统具体由一个工作电极以及一个回路电极构成, 当高频电流在流过了这两个电极之后, 会因为 生理盐水激发生成动态的等离子体, 从而让患者组织内的有机分子键破裂, 并使接触的组织发生气化, 在深层组织部 位还能够形成0.5 1.0mm的均匀凝固层。

在对前列腺进行了电切处理之后, 会在患者的后腺窝表面焦表面形成一定的焦痂, 对于血液供应进行阻断, 也就 能够很好的避免肿瘤细胞黏附种植等问题发生。此外在采用等离子切割基础来进行手术时, 患者组织表面温度还可以 控制在 $40^{\circ} \mathrm{C} \sim 70^{\circ} \mathrm{C}$, 对于患者的闭孔神经以及阴茎神经也会造成损伤, 使得性功能障碍发生率得到了大幅度的缩短 ${ }^{[3]}$ 。 此外因为前列腺包膜跟增生前列腺组织的阻抗存在有一定的差异性, 也就导致了等离子体的切除效率得到了显著的下 降, 这样也就能够为手术人员带来良好的反应时间, 促使手术安全性得到进一步的提升。为了保障手术的顺利进行, 在具体手术过程之中还需要充分注意以下几点: (1)在进行膀脂肿瘤的切割过程中需要进行深人的有效控制, 这样也就 能够避免尿液外渗所导致的感染问题发生, 以保障患者的预后效果。(2如果前列腺增生情况已经严重影响到了膀胱肿 瘤的切除事业, 还需要先进行甲状腺中叶的切除工作, 在手术视野清晰之后方能够进行膀胱肿瘤的切除处理, 保障后 
续手术的顺利进行。(3)在术中需要做好止血工作, 术后进行常规硬模外持续镇痛泵进行止痛处理, 来楲少膀胱痉金的 发生可能性, 并能够有效避免术后继续出血等问题发生。(4)对于一些多发膀胱肿瘤患者, 需要对顶部以及前壁等比较 难操作部位的肿瘤进行切除处理, 随后再处理一些容易操作部位的肿瘤, 这样也就能够始终保持以良好的视野, 来为 后续手术的顺利进行奠定良好的基础 ${ }^{[4]}$ 。(5)术后常规留置导尿管, 随后定期进行膀胱灌注化疗工作, 来让患者的肿瘤 复发机率得到大幅度的降低。(6)在对一些高龄高危患者行手术治疗时, 还要在结合患者具体病情的基础上来进行手术 模式的合理选择, 而不是强求采用同期手术来进行治疗。在具体治疗过程中能够充分重视上述几点, 并进行手术术式 的合理选择，才能够起到良好的前列腺增生合并膀胱癌治疗效果，对于术后复发情况也能够进行有效的控制，借此来 获得良好的治疗效果。

在本次研究中, 所有患者的术后前例腺症状指标均要显著优于手术前, $P<0.05$ 差异有统计学意义。此外 64 例膀 胱癌合并前列腺增生患者中, 仅仅出现了4例并发患者, 而且复发位置均位于前列腺窝以及后尿道中。综上所述, 通 过同期经尿道等离子电切术能够对膀胱癌合并前列腺增生患者起到良好的治疗效果, 其治疗安全性高, 值得临床应用 推广。

\section{参考文献:}

[1]张文栋.同期经尿道等离子电切术治疗膀胱癌合并前列腺增生的临床价值评析 [J].中国医药指南,2020,14(16):113.

[2]李碧君, 单炽昌, 王在盛, 叶亲永.同期经尿道电切术治疗膀胱癌合并良性前列腺增生的临床分析[J]. 中国肿瘤临床 与康复,2020,18(02):168-171.

[3] 陈智林, 李健.同期经尿道电切术治疗浅表性膀胱癌合并前列腺增生的临床效果观察 [ J]. 中国医药科 学, 2020,4(02):178-180.

[4]袁其华,冯秀丽.同期行经尿道电切术治疗膀胱癌并前列腺增生33例临床分析 [J].中国民康医学,2020,11(8):109-113. 